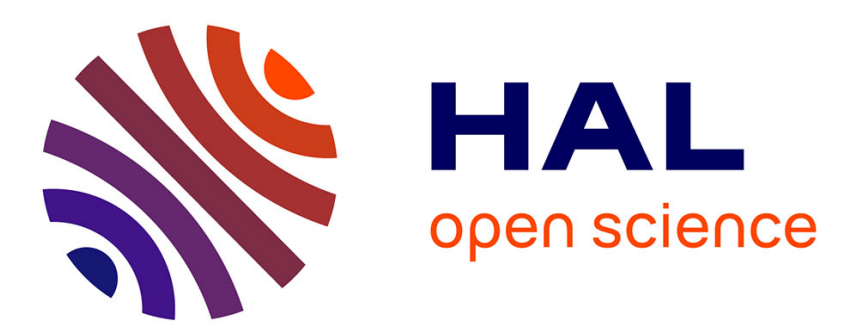

\title{
Techniques pour une gestion multifonctionnelle durable des forêts de montagne: cas des Alpes en France et en Italie.
}

\author{
D. Leclerc, Murièle Millot
}

\section{- To cite this version:}

D. Leclerc, Murièle Millot. Techniques pour une gestion multifonctionnelle durable des forêts de montagne: cas des Alpes en France et en Italie.. Revue forestière française, 1998, 50 (sp), pp.67-81. $10.4267 / 2042 / 5583$. hal-03510465

\section{HAL Id: hal-03510465 \\ https://hal.science/hal-03510465}

Submitted on 4 Jan 2022

HAL is a multi-disciplinary open access archive for the deposit and dissemination of scientific research documents, whether they are published or not. The documents may come from teaching and research institutions in France or abroad, or from public or private research centers.
L'archive ouverte pluridisciplinaire HAL, est destinée au dépôt et à la diffusion de documents scientifiques de niveau recherche, publiés ou non, émanant des établissements d'enseignement et de recherche français ou étrangers, des laboratoires publics ou privés. 


\section{ChAPITRE III}

\section{SYLVICULTURE ET AMÉNAGEMENT DES FORÊTS DE MONTAGNE}

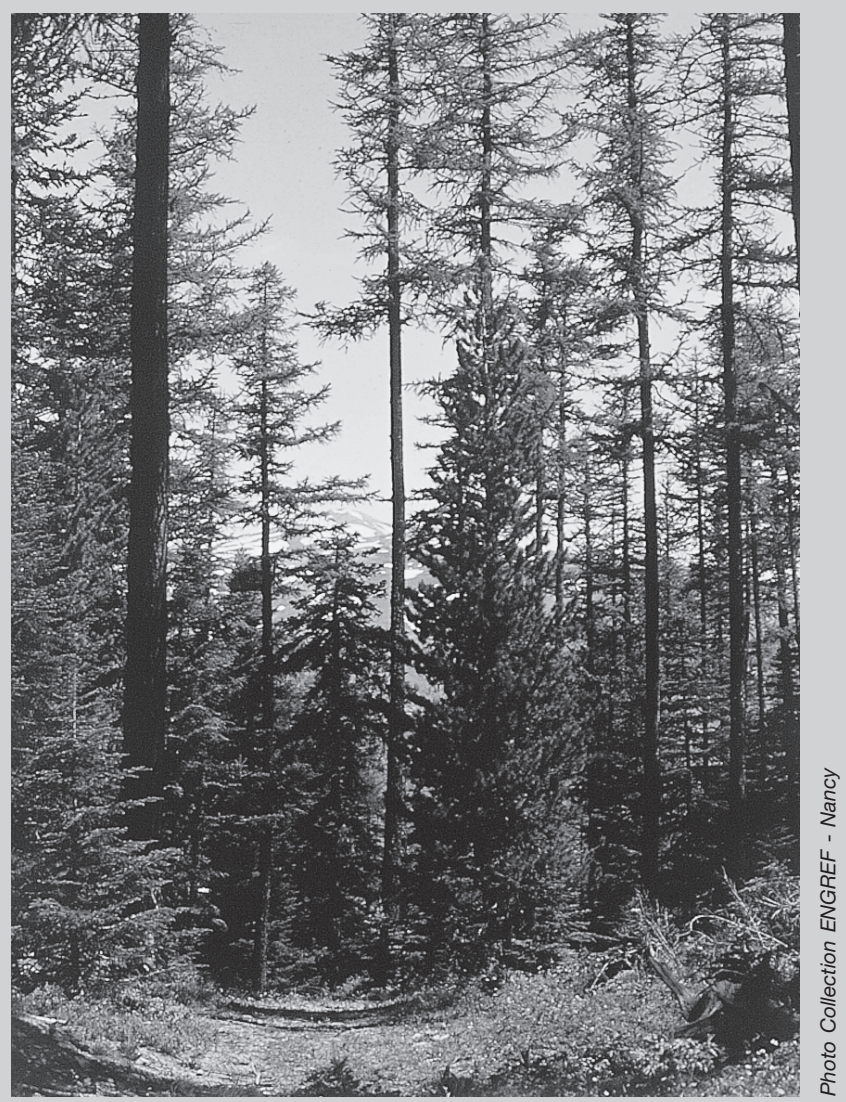





\title{
TECHNIQUES POUR UNE GESTION MULTIFONCTIONNELLE DURABLE DES FORÊTS DE MONTAGNE : CAS DES ALPES EN FRANCE ET EN ITALIE
}

\author{
D. LECLERC - Murièle MILLOT
}

On reconnaît de nos jours à la forêt de montagne de nombreux rôles d'utilité publique, tant du point de vue environnemental (écologie, paysage, patrimoine) que socio-économique (production et protection physique). Cette prise de conscience s'est exprimée notamment dans la résolution $n^{\circ} 4$ de la conférence ministérielle pan-européenne de Strasbourg (décembre 1990) et dans le protocole "Forêt de montagne" inscrit dans la "Convention alpine" discutée à Salzburg (novembre 1991).

Les différentes fonctions assignées à la forêt de montagne ne peuvent être remplies qu'en assurant une gestion adaptée. Celle-ci consiste en une série d'interventions destinées à conserver ou à améliorer la structure des peuplements forestiers. Ces interventions ont un intérêt à la fois sylvicole et économique. Or, on constate de plus en plus l'existence de coupes "à bilan économique négatif", où les interventions sylvicoles jugées indispensables ne peuvent être effectuées que par le biais de modes d'exploitation très onéreux ou ne peuvent pas être réalisées du tout.

II est donc apparu nécessaire d'aborder de façon globale les techniques de conduite de ces peuplements forestiers de montagne et leur exploitation, afin de déterminer les moyens les plus rationnels à mettre en œuvre pour permettre à ces peuplements de remplir leurs fonctions multiples.

\section{PRÉSENTATION DE L’ÉTUDE}

Cet article rend compte d'une étude sur les «Forêts de montagne: techniques pour une gestion multifonctionnelle durable dans les Alpes françaises et italiennes" (1), conduite par des spécialistes français et italiens de la sylviculture et de l'exploitation forestière. II s'est agi d'une opération de démonstration sur six chantiers pilotes (trois en France et trois en Italie).

(1) Étude réalisée dans le cadre du programme LIFE n 94/F/A15/EU/00173 associant : pour la France : le CEMAGREF de Grenoble, I'ONF (Section technique interrégionale et Direction régionale Rhône-Alpes) et le CTBA; pour l'Italie: I'ISAFA (Istituto per l'Assestamento Forestale e per l'Alpicoltura) de Trento (sylviculture), l'ITL (Istituto per la Tecnologia del Legno) de Trento (techniques d'exploitation) et l'IRL (Istituto per la Ricerca sul Legno) de Florence (techniques d'exploitation); pour la Suisse, les contacts ont été surtout avec l'Institut fédéral de Recherches sur la Forêt, la Neige et le Paysage de Birmensdorf. 


\section{LECLERC - Murièle MILLOT}

Le but de l'étude était d'obtenir, sur chacun des sites, le meilleur compromis possible entre objectifs sylvicoles et contraintes d'exploitation, grâce à une expertise commune entre spécialistes de ces diverses disciplines de chaque pays.

\section{Méthodologie}

Les relations entre sylviculture et exploitation dans chaque site ont été identifiées (figure 1, ci-dessous) en distinguant les contraintes à caractère physique, des contraintes à caractère technique (sylviculture, exploitation) et de celles à caractère économique (bilan des coupes).

$1^{\text {re }}$ étape : Préciser les principales caractéristiques de la forêt et ses rôles de protection (identifier les enjeux et la stabilité des peuplements).

$2^{\mathbf{e}}$ étape : Mesurer les incidences techniques et économiques.

Déterminer le type de sylviculture souhaitable et établir les possibilités techniques d'exploitation.

$3^{\text {e }}$ étape : Après avoir déterminé le meilleur compromis sylvicultureexploitation dans le contexte local, réaliser des opérations sylvicoles et d'exploitation.

Dresser le bilan technicofinancier (sylviculture et coûts d'exploitation).

Faire la synthèse.
Figure 1

MÉTHODOLOGIE

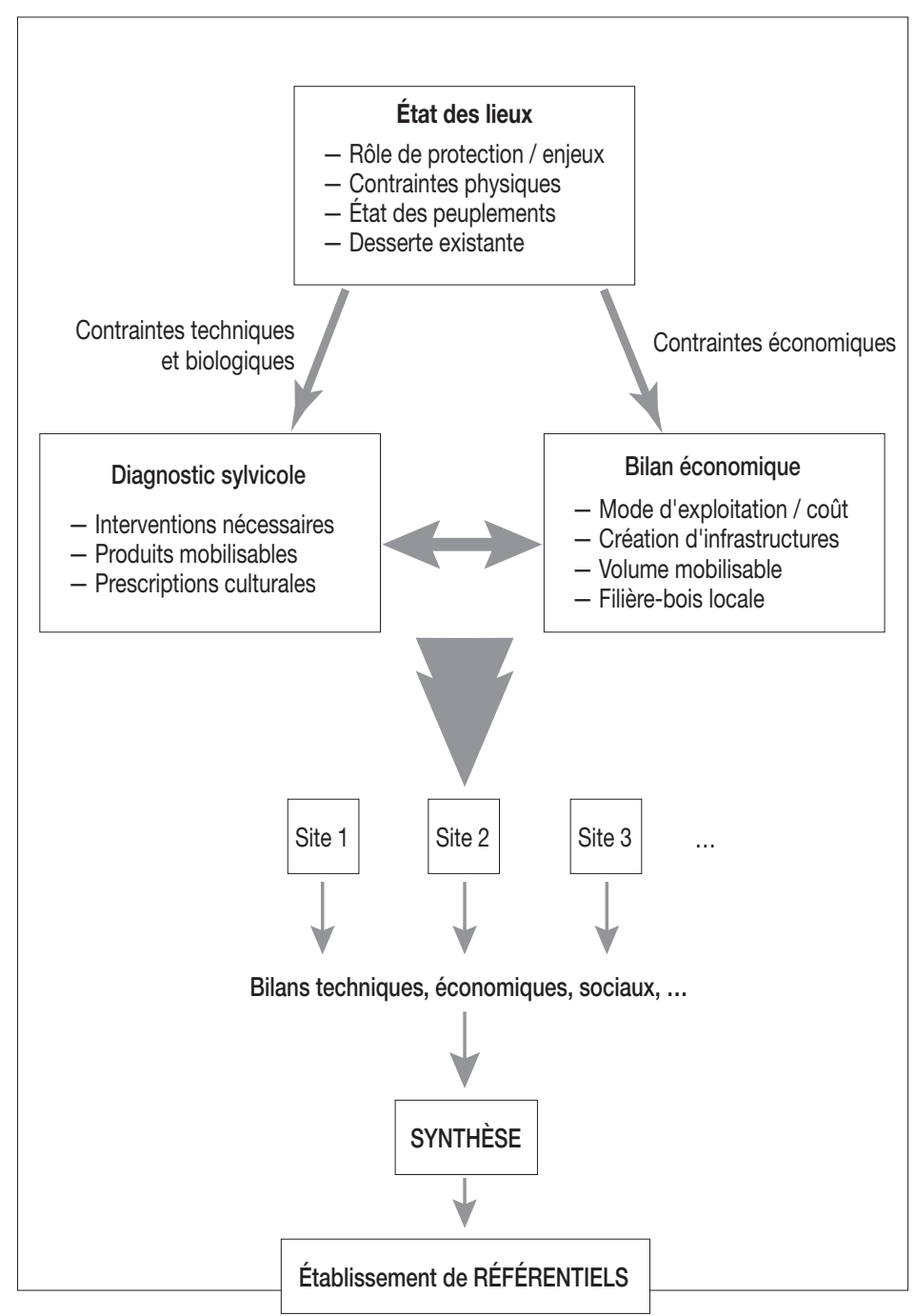




\section{Choix des sites}

Les sites ont été choisis dans des forêts à caractère multifonctionnel marqué, en particulier à fort rôle de protection physique et paysager. Ils sont généralement situés sur des terrains en forte pente, traversés par des sentiers passant à proximité des activités humaines. Hormis le site du Boutat (hêtraie), ils concernent essentiellement des pessières subalpines.

\section{Démarche expérimentale}

- Protocole de description des parcelles

Analyse sylvicole :

- Parcours exhaustif des sites selon un maillage de $50 \mathrm{~m} \times 50 \mathrm{~m}$.

- Description de 25 ares suivant une typologie existante adaptée (STIR-CEMAGREF) et cartographie.

- Mesures dendrométriques sur une placette échantillon de 5 ares.

Analyse du risque :

- Analyse des aléas (avalanches, chute de blocs...).

- Analyse des enjeux menacés (habitations, voies de communication...).

Analyse de la valeur écologique:

- Zonage des grands types de milieux.

- Reconnaissance de la flore ou faune rares (Tétras en particulier) pouvant justifier des précautions particulières.

Analyse paysagère :

- Détermination des zones de forte sensibilité paysagère.

- Importance de la fréquentation.

À partir de ces éléments, ont été établis des diagnostics par site définissant des règles d'intervention sylvicoles optimales a priori. La méthode d'exploitation a été choisie en confrontant les problèmes d'exploitation avec ces règles sylvicoles, en vue de maximiser le bilan économique en fonction des contraintes techniques. Puis, les coupes prévues à partir de ces diagnostics ont été martelées, vendues et exploitées.

\section{- Protocole d'étude de l'exploitation forestière}

Les opérations d'exploitation forestière ont été intégralement suivies et analysées.

Mesure des possibilités techniques:

- Configuration de la desserte existante.

- Caractérisation des matériels utilisés et installations nécessaires (câble en particulier).

- Chronométrage des différentes opérations élémentaires (bûcheronnage, débusquage et débardage).

- Cubage des arbres exploités.

- Mesure des distances de débusquage et de débardage.

- Volume récolté (total et /ha).

Mesure des impacts de l'exploitation sur l'environnement:

- Mesure et relevé des dégâts de l'exploitation sur le peuplement restant et sur le terrain.

- Description du milieu après exploitation. 


\section{LECLERC - Murièle MILLOT}

Dépouillement et traitement des données :

Les principaux indicateurs technico-économiques ont été déterminés pour chaque type de matériel utilisé pour préciser leurs limites d'utilisation.

\section{ÉTUDE DES SITES}

\section{Les sites français}

Tous les sites sont localisés dans les Alpes du Nord. L'étude de l'un des trois premiers sites pressentis concernant la forêt communale d'Abondance (Haute-Savoie) n'ayant pu être menée à terme dans le cadre de ce programme pour des raisons développées plus loin, un site supplémentaire a donc été trouvé pour le remplacer dans la forêt communale des Contamines-Montjoie (HauteSavoie).

- Site du Boutat (30 ha)

Forêt domaniale du Boutat (Isère) : parcelles 7 et, pour partie, 41, 42 et 43.

Cette zone, située sur le rebord Est de la Chartreuse, vient buter contre les falaises urgoniennes du massif, hautes de 200 à $300 \mathrm{~m}$. Elle appartient à la série de la hêtraie calcicole à acidicline (altitude comprise entre 1100 et $1500 \mathrm{~m}$ ). La futaie dense de hêtres à bois moyens qui la constitue joue un rôle de protection efficace contre les chutes de blocs des falaises et, un peu moins, contre les avalanches. Située en périphérie du Parc naturel régional de Chartreuse, elle joue un rôle paysager très perceptible depuis la vallée de l'Isère.

Diagnostic sylvicole

L'analyse typologique des peuplements fait apparaître cinq groupes structuraux différents (125 placettes inventoriées) :

groupe 1: Hêtraie pure régularisée dense à très dense à petits bois (767 tiges/ha).

groupe 2 : Futaie irrégulière mélangée Hêtre-Épicéa (483 tiges/ha).

groupe 3 : Hêtraie régularisée à bois moyens ouverte en cours de rajeunissement (427 tiges/ha).

groupe 4 : Hêtraie régularisée à bois moyens fermée (487 tiges/ha).

groupe 5 : Futaie irrégulière très claire à petits bois (540 tiges/ha).

Directives de martelage

Pour préserver le paysage et le rôle de protection des peuplements, il faut irrégulariser la forêt en isolant des bouquets et en ouvrant des plages de 5 à 20 ares. Au total, 1523 tiges ont été martelées pour un volume commercial total de $819 \mathrm{~m}^{3}$.

Diagnostic d'exploitation

La configuration de la coupe (1000 m de large pour $500 \mathrm{~m}$ de haut) a nécessité l'utilisation d'un câble-mât mobile en l'absence de desserte sur le versant. L'utilisation du câble-mât a permis de réaliser cette opération en concentrant les interventions dans trois zones (14 ha) avec un prélèvement élevé supérieur à $200 \mathrm{~m} 3 /$ ha, minimum nécessaire pour une exploitation rentable par câble, un compromis devant être trouvé entre contrainte économique et objectif d'irrégularisation. Cinq lignes ont été matérialisées (400 $\mathrm{m}$ de long chacune), permettant de dégager les régénérations acquises dans le groupe 4 par des ouvertures en forme de "doigt de gant" et de pratiquer des coupes "secondaires" pied à pied dans le groupe 3 et des coupes d'éclaircie et d'ensemencement par plage dans le groupe 1 (voir figure 2, p. 71). Dans le cadre de ce programme, seules les lignes de câble 1 et 2 , correspondant à la parcelle 7 la plus riche, ont été installées et étudiées minutieusement. 


\section{Exploitation}

Sur ces deux lignes, $396 \mathrm{~m}^{3}$ ont été exploités. Les temps de travail réellement productif sont en dessous de $30 \%$, ce qui est un résultat assez passable pour cette technique. L'intervention a été globalement bien intégrée, même si la neige accentue l'impact des lignes de câble et fait ressortir les "doigts de gant" (voir photographie, p. 75).

L'exploitation a coûté au total $230 \mathrm{~F} / \mathrm{m}^{3} \mathrm{HT}$ et les bois vendus façonnés ont rapporté $260 \mathrm{~F} / \mathrm{m}^{3} \mathrm{HT}$, ce qui constitue un léger bénéfice pour cette forêt de protection. Cet équilibre est fragile, la marge du câbliste étant elle-même faible. Mais, en accentuant au besoin les interventions aux altitudes supérieures sur hêtraie à petits bois (groupe $n^{\circ} 1$ ), interventions les plus coûteuses du point de vue de l'abattage comme du débardage, l'opération doit pouvoir être reproduite sur les parcelles voisines de configuration identique dans l'immédiat et, dans le même versant, d'ici une trentaine d'années.

Figure 2 EXPLOITATION SUR LE SITE DU BOUTAT (Isère) : OUVERTURES AUTOUR DES LIGNES DE CÂBLE

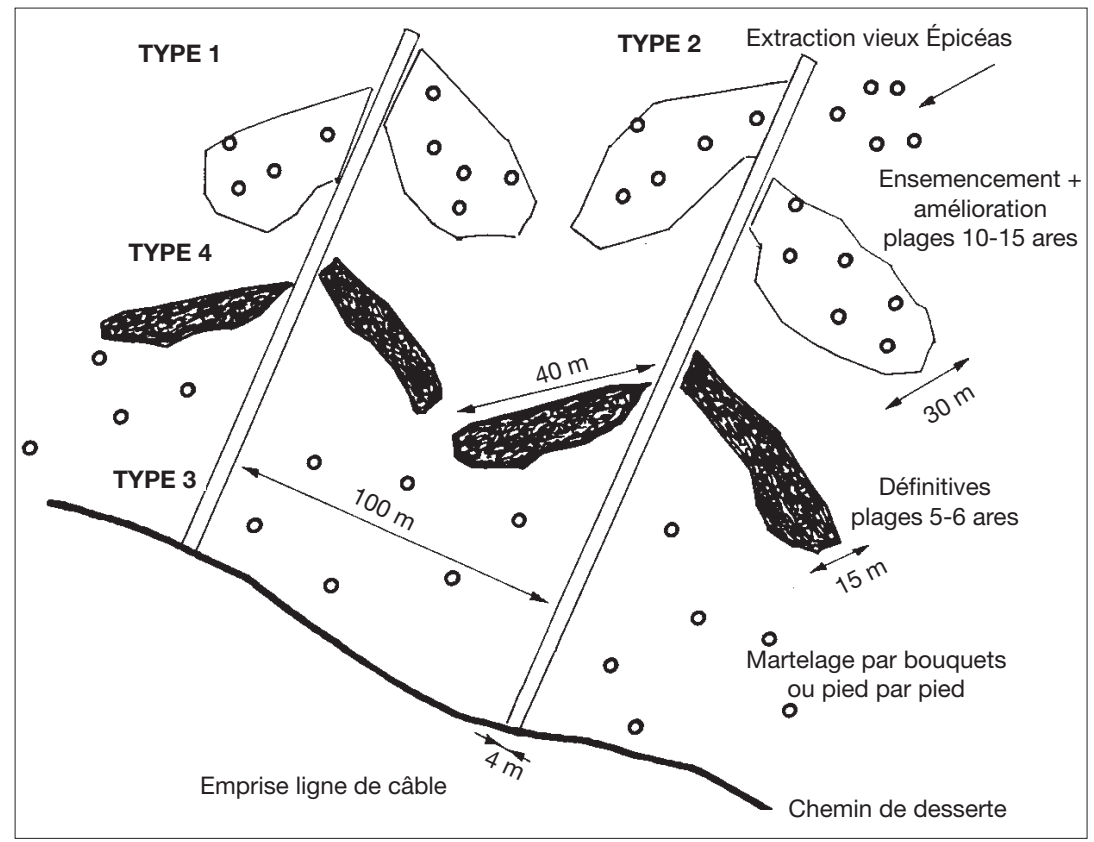

- Site de Pralognan (13 ha)

Forêt communale de Pralognan-la-Vanoise (Savoie) : Massif de l'Isertan : parcelles 37, 39 et 40.

Cette zone située dans la vallée de la Tarentaise est aux portes du Parc national de la Vanoise. Elle appartient à la série de la pessière subalpine mésophile (altitude comprise entre 1450 et $1750 \mathrm{~m}$, exposition Nord-Nord-Ouest). Avant les tempêtes de 1968 et 1977 qui l'ont dévastée, elle était constituée essentiellement d'un peuplement dense et régularisé d'Épicéa dont il ne reste actuellement que des bouquets plus ou moins complets. Hormis son rôle de protection contre les avalanches et les chutes de pierres menaçant les infrastructures touristiques très nombreuses à 


\section{LECLERC - Murièle MILLOT}

Pralognan (station de ski en hiver et station d'été), sa situation dans l'axe de la Vallée du Doron de Pralognan lui confère une sensibilité paysagère forte. Par ailleurs, elle abrite la Linnée boréale (Linnaea borealis L.), espèce très rare et protégée par la convention alpine.

Diagnostic sylvicole

L'analyse typologique des peuplements fait apparaître trois zones (voir figure 3, p. 73), une zone basse constituée de futaie vieillie d'Épicéa plus ou moins ouverte, une zone médiane multi-strate plus irrégulière, et une zone haute dominée par des types régularisés à bois moyens, l'ensemble étant encore riche en bois $\left(300 \mathrm{~m}^{3} / \mathrm{ha}\right)$.

Directives de martelage

Le maintien de peuplements complets en limitant les ouvertures de 5 à 10 ares, la recherche d'un meilleur étagement ainsi que l'ouverture à la lumière des semis acquis ont été décidés. 439 tiges ont été martelées pour un volume commercial total de $820 \mathrm{~m}^{3}$.

Diagnostic d'exploitation

Toute infrastructure routière étant exclue, c'est l'option câble long qui a été retenue. Deux lignes de câble ont été posées, l'une de $580 \mathrm{~m}$ de long, l'autre de $920 \mathrm{~m}$. Elles desservent les deux bordures de la zone étudiée, oubliant un peu la zone centrale. Pour des raisons financières, une troisième ligne plus centrale n'a pu être installée. Les lignes ont été repérées de façon à permettre l'ouverture de fentes dans les bois moyens du haut et la récolte sanitaire dans les gros bois du bas.

\section{Exploitation}

Ces deux lignes limitent l'impact paysager de la coupe dans cette région très touristique en été comme en hiver, et permettent d'exploiter jusqu'à $50 \mathrm{~m}$ de part et d'autre de l'axe de ces lignes. $743 \mathrm{~m}^{3}$ ont été exploités. Les bons rendements observés sont à mettre en relation avec le volume de l'arbre moyen prélevé et le volume total exploité par ligne $\left(223 \mathrm{~m}^{3}\right.$ sur la ligne 1 et $520 \mathrm{~m}^{3}$ sur la ligne 2). L'exploitation a coûté $295 \mathrm{~F} / \mathrm{m}^{3} \mathrm{HT}$ et les bois vendus façonnés ont rapporté $388 \mathrm{~F} / \mathrm{m}^{3} \mathrm{HT}$, soit un bénéfice substantiel de $93 \mathrm{~F} / \mathrm{m}^{3} \mathrm{HT}$ pour la commune.

\section{- Site des Contamines-Montjoie (25 ha)}

Forêt communale des Contamines-Montjoie (Haute-Savoie) : parcelles 37 et 47.

Cette zone située à la limite entre le massif du Mont-Blanc et le massif du Beaufortain couvre le versant Est abrupt de la vallée étroite du Bon Nant. Elle appartient à la série des pessières acidiphiles (altitude de 1500 à $1970 \mathrm{~m}$ ) sur des pentes comprises entre 50 et $70 \%$. Elle est constituée sur plus de $60 \%$ de sa surface d'une vieille futaie régularisée d'Épicéa. Des couloirs d'avalanches qui traversent cette forêt lui confèrent un rôle de protection très actif. La proximité du massif du Mont-Blanc attire aussi un grand nombre de touristes en été comme en hiver, qui utilisent l'important réseau de sentiers traversant cette forêt ainsi qu'une ancienne voie romaine en contrebas.

Diagnostic sylvicole

Le site étudié se décompose en trois zones distinctes : en altitude $>1600 \mathrm{~m}$, une futaie très claire ; un peuplement de première génération $(<100$ ans) ; la partie la plus importante en futaie régulière âgée et dense $\left(>250 \mathrm{~m}^{3} / \mathrm{ha}\right.$ ) sans régénération suffisante.

Directives de martelage

Les interventions préconisées sont destinées à pérenniser le couvert forestier, donc à dégager le peu de régénération présente et initier un renouvellement naturel, maintenir la stabilité des arbres dominants en travaillant par bouquets et par l'ouverture de fentes obliques par rapport à la pente 
Figure 3 SITE DE PRALOGNAN-LA-VANOISE (Savoie)

Cartographie des structures de peuplements

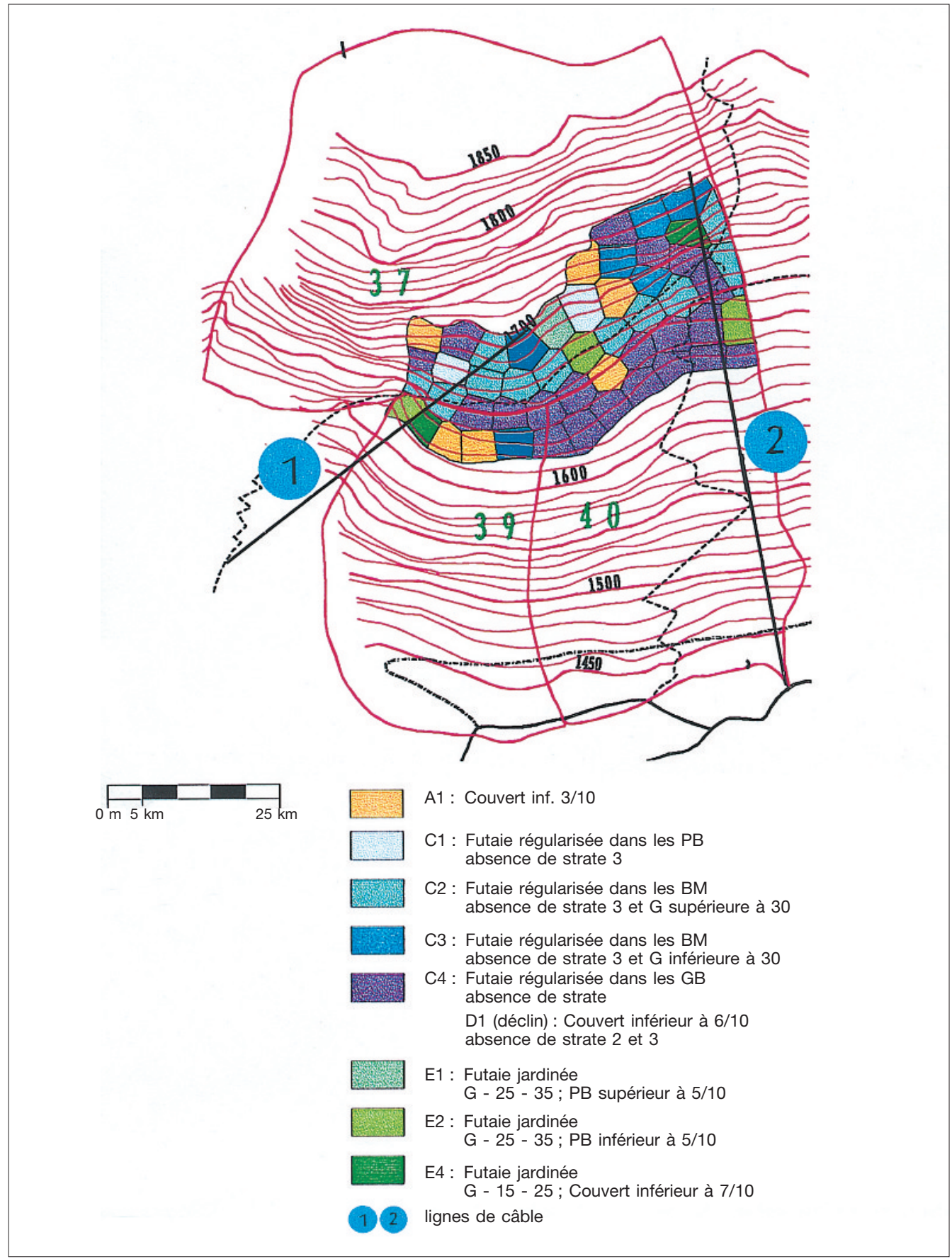

Carte réalisée sur Arc Info par E. Mermin - Division EPM - CEMAGREF Groupement de Grenoble. 


\section{LECLERC - Murièle MILLOT}

pour ne pas créer de couloirs d'avalanches ou de chutes de pierres, et exploiter les bois mûrs et surannés. 615 tiges ont été martelées pour un volume commercial total de $1397 \mathrm{~m}^{3}$.

Diagnostic d'exploitation

L'association originale du câble longue portée et du tracteur a été choisie. En effet, le débardage du site aurait pu se faire théoriquement en utilisant l'ancienne voie romaine, mais l'accès aux tracteurs est interdit sur certains tronçons. Le skidder n'a donc été utilisé que pour le débusquage et c'est le câble longue portée qui a pris le relais au-dessus de cette voie.

\section{Exploitation}

La coupe vendue en bloc et sur pied a rapporté à la commune $94 \mathrm{~F} / \mathrm{m}^{3} \mathrm{HT}$. Elle a coûté à l'entreprise qui l'a achetée $75 \mathrm{~F} / \mathrm{m}^{3} \mathrm{HT}$ d'abattage et $300 \mathrm{~F} / \mathrm{m}^{3} \mathrm{HT}$ de débardage.

\section{- Site d'Abondance (15 ha)}

Forêt communale d'Abondance (Haute-Savoie) : parcelles 16 et 57.

Cette zone est située dans le massif du Chablais dans la vallée touristique d'Abondance. Elle correspond à l'étage montagnard des Alpes externes (altitude moyenne $1300 \mathrm{~m}$, exposition Sud-Est). Surplombant le village et son abbaye renommée, son rôle de protection contre la chute de blocs, qui les menacent directement, est très important. Toute intervention doit également avoir impérativement un impact paysager limité jusqu'à la plage à dépôt en aval.

Diagnostic sylvicole

L'analyse typologique des peuplements fait apparaître trois zones: une partie haute de pessière jeune et très dense, une zone centrale importante régularisée en gros bois, et une partie inférieure jardinée riche en Hêtre. La régénération est globalement déficitaire sur l'ensemble.

Directives de martelage et diagnostic d'exploitation

Les parties supérieure et centrale sont inaccessibles. Seul un hélicoptère gros porteur peut intervenir. Il a donc été préconisé une intervention forte avec période de rotation élevée (30 ans) avec extraction systématique des très gros bois pour mettre en lumière le sol afin de favoriser la régénération naturelle. Dans la partie inférieure, accessible au tracteur, les épicéas dépérissants ont été martelés ainsi que les hêtres à "gros houppiers" pour éclairer la régénération. Une surface terrière suffisamment élevée en Hêtre a été laissée afin de maintenir au peuplement un rôle de protection optimale contre les chutes de blocs et pour l'aspect paysager. Par ailleurs, ces hêtres ont encore une bonne valeur économique. 1232 tiges ont été martelées pour un volume commercial total de $1457 \mathrm{~m}^{3}$.

\section{Exploitation}

Elle n'a pu avoir lieu ! L'exploitation préconisée par hélicoptère pour le haut et l'obligation de poser des filets contre les chutes de pierres pour la protection de la route située en contrebas rendent la coupe déficitaire. La commune, peu armée pour prendre la responsabilité d'importants travaux perturbants à court terme, s'est opposée dans un premier temps à cette réalisation déficitaire. Une aide à l'exploitation forestière dans les zones difficiles dans le cadre du programme "Compétitivité Plus" a été sollicitée, mais n'a pas été obtenue. L'accord enfin obtenu de la part de la commune est arrivé trop tard pour suivre ce site dans le cadre de ce projet LIFE. L'exploitation aura quand même bien lieu ultérieurement. Cette intervention indispensable pour le maintien du rôle de protection de cette forêt s'est donc limitée au martelage. C'est un cas malheureusement typique illustrant un problème structurel : la prise en compte du long terme justifie une intervention financière complémentaire et la prise de risques à court terme (déstabilisation possible de blocs pendant l'exploitation) qu'une municipalité ne peut pas facilement assumer seule. 


\section{Site du Boutat après exploitation.}

Photo E. MERMIN

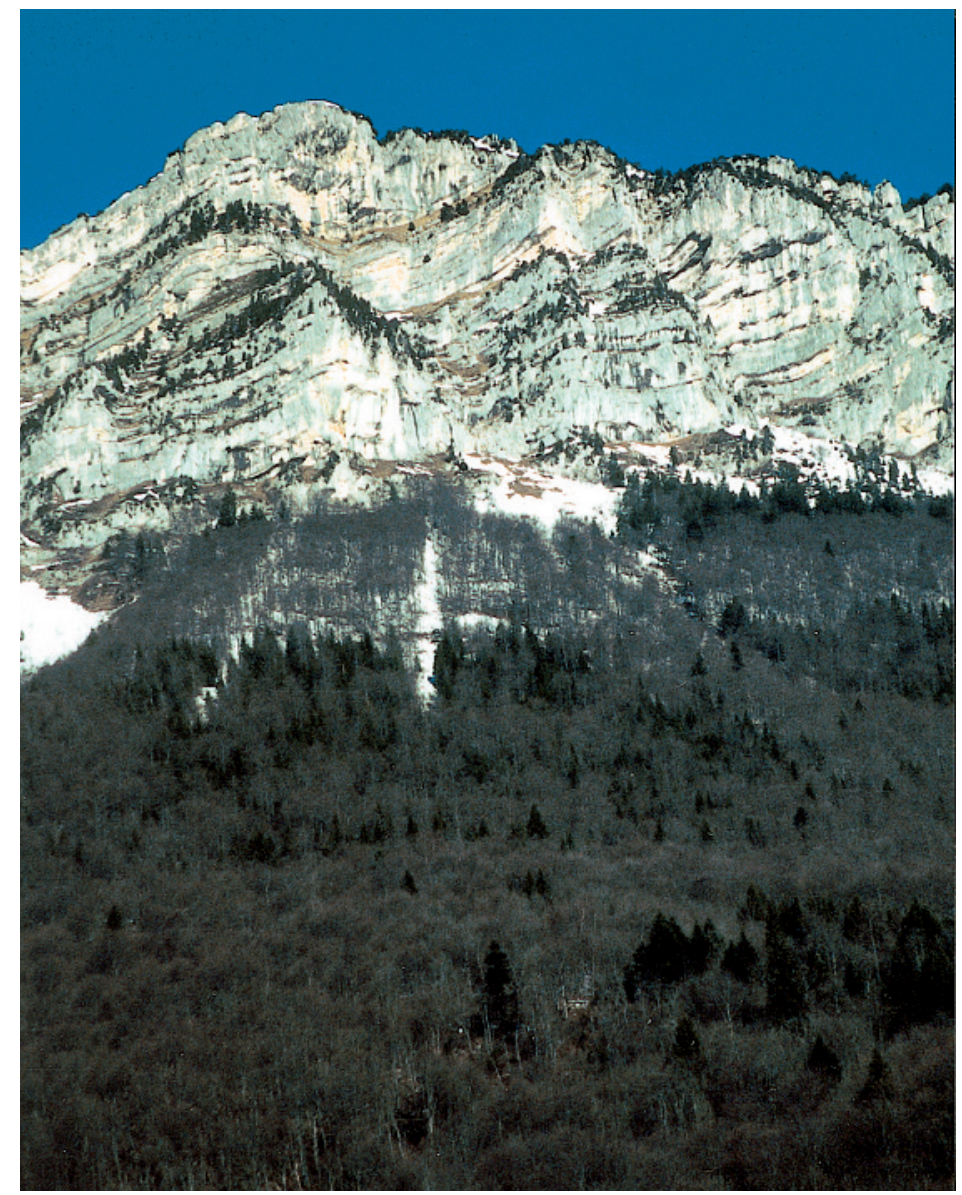

\section{Les sites italiens}

Les trois sites sont situés dans la province autonome du Trentin, région très montagneuse entre le lac de Garde et l'Autriche et couverte de forêts pour plus de 54 \% du territoire. Plus précisément, ils sont localisés dans le Val di Fiemme et ont la particularité d'appartenir tous à la même collectivité depuis le Moyen-Âge : la Magnifica Comunità di Fiemme, qui assure elle-même l'exploitation, le débardage et surtout la transformation des bois (scierie) avec ses propres personnels. La Comunità utilise régulièrement des câbles, mais plutôt pour des "coupes de lisières" (coupes en bande le long de l'axe du câble) en futaie régulière.

\section{- Site de Cadino (26 ha)}

Forêt de la Magnifica Comunità di Fiemme, commune de Castello di Fiemme, lieu de Costa dei Casoni : parcelle 25.

Cette zone, située sur le versant Sud-Ouest de la vallée du Cadino, est très pentue (altitude de 1650 à $1900 \mathrm{~m}$ ). Elle est constituée de peuplements d'Épicéa soit purs, soit, par endroits, mélangés avec du Mélèze. Elle protège des refuges et des chalets, ainsi que la route du fond de la vallée, contre les avalanches et les chutes de pierres. Elle maintient aussi la stabilité du versant par son rôle sur les infiltrations d'eau. 


\section{LECLERC - Murièle MILLOT}

\section{Diagnostic sylvicole}

L'analyse structurale des peuplements fait apparaître deux zones : la zone la plus haute régularisée et vieillie $(77 \%)$ et la zone plus basse constituée de peuplements multistratifiés. La régénération naturelle est très peu abondante $(<10 \%$ en surface) et le peuplement très instable à cause de son âge avancé et de sa régularité. De petites trouées dues à des chablis ou des attaques d'insectes sont aussi observées.

Directives de martelage

Il est apparu urgent de régénérer (artificiellement même s'il le faut) le Mélèze et le Pin cembro. Trois ouvertures en trouées ont été décidées dans la partie la plus haute, et les arbres surannés ont été prélevés partout. 159 tiges ont été martelées pour un volume commercial total de $343 \mathrm{~m}^{3}$.

\section{Figure 4 RÉPARTITION DES CLASSES DE DIAMÈTRES (CM) DES BOIS MARTELÉS À CADINO}

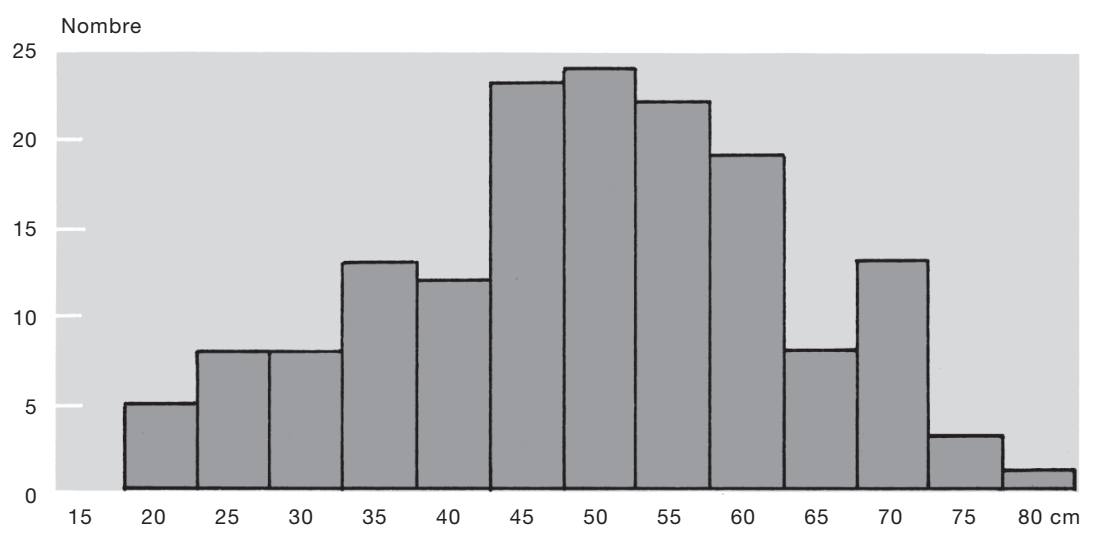

\section{Diagnostic d'exploitation}

Cette opération, urgente à cause de la trop grande maturité et régularité des peuplements en place, a été facilitée par la construction d'une nouvelle route forestière qui permet d'accéder facilement au massif. Les trois ouvertures se trouvent à proximité de cette route. L'opération sera poursuivie dans les années à venir par l'utilisation d'un câble-mât installé sur cette même route.

\section{Exploitation}

L'abattage-façonnage et le débardage par tracteur agricole équipé d'un câble ont été exécutés à partir de cette route. Les bois exploités en bois courts ont été tirés vers le haut (distance $>50 \mathrm{~m}$ ).

\section{- Site de Sadole (51 ha)}

Forêt de la Magnifica Comunità di Fiemme, commune de Ziano di Fiemme, lieu du Corona di Sadole : parcelle 40.

Cette zone très pentue, sur le versant Ouest de la vallée de Sadole, est essentiellement située dans la série de la pessière à Myrtille subalpine (altitude de 1420 à $1865 \mathrm{~m}$ ). Elle est constituée principalement de peuplements purs d'Épicéa. Elle joue un rôle de protection contre les chutes de blocs sur $13 \%$ de sa surface. Elle est également très fréquentée en été par des promeneurs qui utilisent un important réseau de sentiers. 


\section{Sylviculture et aménagement des forêts de montagne}

\section{Diagnostic sylvicole}

L'analyse structurale des peuplements fait apparaître trois zones : la partie supérieure est une pessière subalpine en bouquets denses et très âgés, la partie Sud-Est est multistratifiée et la partie intermédiaire est aussi assez mûre (gros bois importants très présents) mais plus irrégulière (à proximité de blocs rocheux). L'Épicéa représente $95 \%$ du nombre total de tiges. $84 \%$ des tiges présentent des défauts (vent, neige, blessures dues aux pierres). La régénération est assez présente par petites trouées ( $23 \%$ de la surface en moyenne).

\section{Directives de martelage}

II est prévu de commencer la phase de régénération en exploitant au total sur le massif $3500 \mathrm{~m}^{3}$ sous forme de petites trouées dans les peuplements mûrs et dans la partie plus multistratifiée (SudEst), pour ne réaliser qu'une seule intervention bénéficiaire sur l'ensemble du site à chaque passage en coupe. 383 arbres ont été martelés pour un volume commercial total de $1070 \mathrm{~m}^{3}$.

\section{Diagnostic d'exploitation}

La forme de la parcelle, très longue dans le sens de la pente et relativement éloignée de la route la plus proche, a influencé le choix d'exploitation par câble suivant deux lignes sur 2,5 ha au total. II s'agit en pratique de deux coupes rases par bandes, mais avec un découpage soigné des lisières selon la structure en bouquets du peuplement existant. Certains bouquets ont été maintenus le long de la ligne ou même sur la ligne pour casser l'effet d'ouverture.

\section{Exploitation}

Très peu de dégâts ont été constatés sur les arbres et sur le sol. Cette coupe a été largement bénéficiaire pour la communauté. On peut juger, d'après la photographie prise après coupe (photo 2, cidessous), de la réussite sur le plan paysager de cette opération plutôt brutale, mais ayant tiré parti d'une structure favorable des peuplements. Cette opération est reproductible dans le temps, bande par bande, sur l'ensemble de la parcelle.

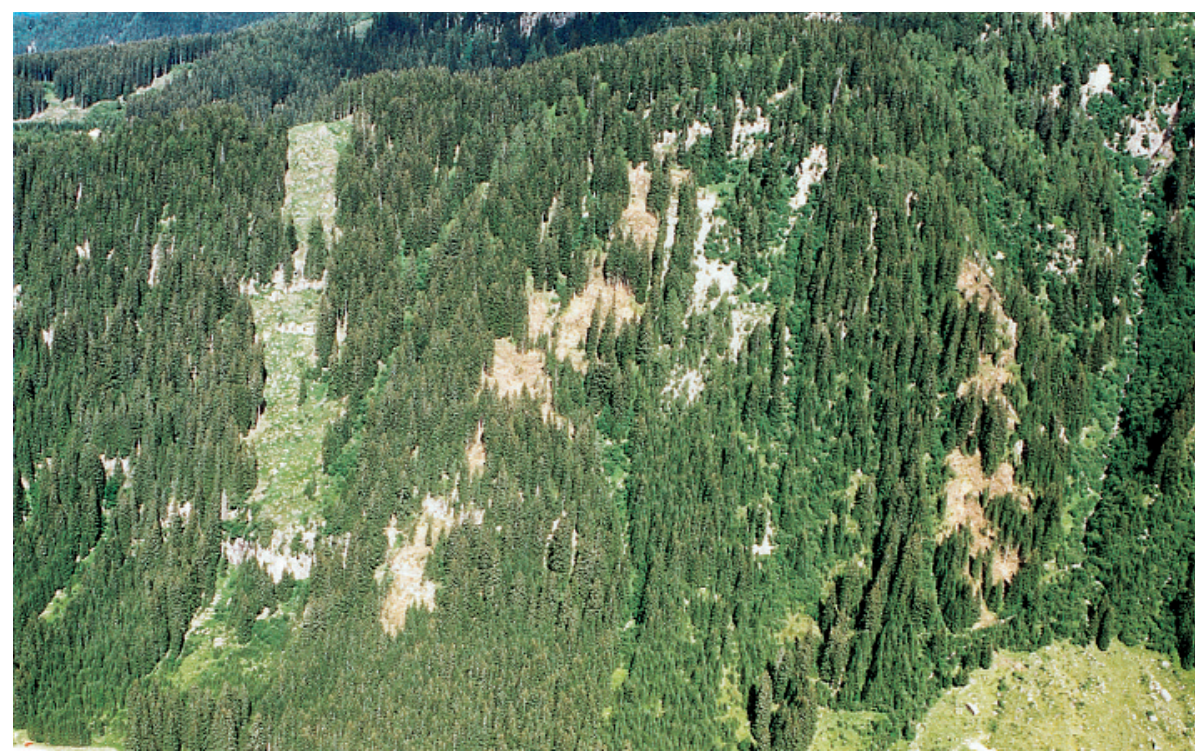

Site de Sadole après exploitation.

Photo Vittorio TOSI - ISAFA TRENTO 


\section{LECLERC - Murièle MILLOT}

- Site de Lavazè (31 ha)

Forêt de la Magnifica Comunità di Fiemme, commune de Varena, lieu de Palua-Malga Ora : parcelles 55 et, pour partie, 54 et 56 .

Cette zone très ouverte et peu pentue relie les cols de Lavazè et d'Ocolini (altitude de 1790 à 1890 m). Au niveau de la "zone de combat", en pessière subalpine, elle est constituée essentiellement de peuplements d'Épicéa (70-80 \%) et de Pin cembro. Elle est située dans une zone très touristique dont elle est un élément important, notamment pour le ski de fond, et abrite également une faune intéressante (ongulés et oiseaux).

Diagnostic sylvicole

L'analyse structurale des peuplements révèle deux zones : un peuplement pur d'Épicéa monostrate et un peuplement mélangé d'Épicéa et de Pin cembro. Ce site a un rôle de récréation prépondérant. L'ensemble est assez âgé et régularisé ( $25 \%$ seulement de la surface en peuplement irrégulier pied à pied) sous forme de petites surfaces homogènes (3-4 ares maximum). La régénération naturelle est très insuffisante sur plus de $80 \%$ de la surface.

Directives de martelage

C'est en s'appuyant sur quelques rares taches de régénération existantes qu'ont été marqués les arbres. Le renouvellement des très vieux arbres a été accéléré et trois ouvertures ont été pratiquées. L'objectif de la coupe était de remplacer les vieux bois et de commencer la régénération en augmentant l'arrivée de la lumière au sol tout en gardant une allure paysagère convenable en pratiquant de petites ouvertures. 162 tiges ont été martelées pour un volume commercial total de $278 \mathrm{~m}^{3}$.

Diagnostic d'exploitation

Ce site très accessible et plat a permis l'utilisation de tracteur agricole équipé de câble.

Exploitation

Cette opération a été très bénéficiaire pour la communauté et sera très facilement étendue année par année à l'ensemble de la parcelle. Les dégâts au sol sont insignifiants, bien que $20 \%$ des arbres restants aient été touchés lors du débardage au tracteur.

\section{BILAN}

\section{Prévention des risques naturels}

Les conséquences de l'ensemble de ces opérations sur la stabilité des versants, donc sur le rôle de protection de ces forêts, ne pourront certes être évaluées que dans plusieurs années. On a cependant constaté que l'intervention sur le site de Pralognan n'a pas fragilisé le peuplement: en effet, de violents coups de vent ont traversé la commune peu de temps après l'exploitation sans provoquer de chablis.

\section{Limitation de l'impact paysager}

Dans l'ensemble, le paysage a été correctement sauvegardé, même sur le site de Sadole, où le martelage sous forme de coupe rase pouvait laisser craindre le pire à première vue : en fait, le traitement des lisières en a totalement estompé l'impact. Au Boutat, où la neige tombée quelques mois après les travaux avait accentué la visibilité des lignes de câbles (voir photographie, p. 75), la végétation a masqué toutes les trouées dès le printemps. 


\section{Exploitation}

Le tableau ci-dessous met en parallèle les chiffres correspondant à l'exploitation sur chacun des 6 sites. Le bilan économique fait apparaître l'importance du volume total de la coupe et du volume de l'arbre moyen.

Toute autre comparaison est délicate, compte tenu de la diversité des reliefs et des approches sylvicoles et techniques. La forte rentabilité du débardage par câble long sur le site de Sadole, liée bien sûr à la concentration des coupes, est remarquable. À l'inverse, les résultats très médiocres sur le site du Boutat montrent les conséquences pénalisantes de la mauvaise organisation des chantiers d'exploitation. Les faibles temps de bûcheronnage et de débardage sur les sites de Cadino et de Lavazè résultent de la facilité d'accès, une route traversant chacun de ces sites.

\begin{tabular}{|c|c|c|c|c|c|c|c|c|c|}
\hline Site & $\begin{array}{l}\text { Surface } \\
\text { totale } \\
\text { (ha) }\end{array}$ & $\begin{array}{l}\text { Surface } \\
\text { exploitée } \\
\text { (ha)* }\end{array}$ & $\begin{array}{l}\text { Nombre } \\
\text { de tiges }\end{array}$ & $\begin{array}{l}\text { Volume } \\
\text { martelé } \\
\left(\mathrm{m}^{3}\right)\end{array}$ & $\begin{array}{l}\text { Volume } \\
\text { exploité } \\
\left(\mathrm{m}^{3}\right)\end{array}$ & $\begin{array}{c}\text { Temps } \\
\text { bûcheronnage } / \mathrm{m}^{3} \\
\text { (minutes) }\end{array}$ & $\begin{array}{c}\text { Coût } / \mathrm{m}^{3} \\
\text { en } \mathrm{F}\end{array}$ & $\begin{array}{c}\text { Temps } \\
\text { débardage } \\
\mathrm{m}^{3} \\
\text { (minutes) }\end{array}$ & $\begin{array}{c}\text { Coût } / \mathrm{m}^{3} \\
\text { en F }\end{array}$ \\
\hline Boutat $\ldots \ldots \ldots \ldots$ & 30 & 14 & 1523 & 819 & 395 & 36,5 & 50 & 40,3 & 180 \\
\hline Pralognan .......... & 13 & 13 & 439 & 820 & 743 & 24,9 & 70 & 13,3 & 180 \\
\hline Contamines ......... & 25 & 25 & 615 & 1397 & 1397 & ** & 75 & 9,5 & 300 \\
\hline Cadino ............ & 26 & 9 & 159 & 343 & 343 & 18,0 & 44 & 14,2 & 115 \\
\hline Sadole ............ & 51 & 2,5 & 383 & 1070 & 1070 & 26,0 & 65 & 6,6 & 72 \\
\hline Lavazè & 31 & 0,7 & 162 & 278 & 278 & 11,3 & 28 & 7,8 & 29 \\
\hline
\end{tabular}

* Surface des ouvertures sous forme de trouées réellement pratiquées en Italie.

** Opération prise en cours de route : mesures de bûcheronnage non connues.

\section{Bilan de la méthode}

L'intérêt de la cartographie des parcelles, prenant en compte leur structure (strates) et leur surface terrière, a permis, d'une part de localiser les zones d'instabilité où il faut intervenir rapidement, d'autre part d'identifier les volumes mobilisables par le biais de la surface terrière afin de choisir le mode d'exploitation adapté permettant d'optimiser le prélèvement.

\section{CONCLUSIONS}

Le projet LIFE "Forêt de montagne : outils pour une gestion multifonctionnelle durable" a permis de trouver une issue relativement satisfaisante pour six chantiers délicats, en jouant sur l'adéquation optimale de la sylviculture au mode d'exploitation forestière.

Cela a nécessité une analyse au cas par cas, débouchant à chaque fois sur une solution originale. II n'y a donc pas de recette universelle, mais plutôt une méthode générale, classique à l'échelle de l'aménagement forestier: analyse des fonctions ou attentes sociales, de l'état des peuplements et de leur dynamique, des contraintes technico-économiques, définition d'un type de sylviculture souhaitable, et choix d'une combinaison sylviculture-mode d'exploitation compatible avec tous ces éléments.

Sur les sites italiens, où l'on bénéficiait d'une filière-bois intégrée et de techniques d'exploitation variées, la réflexion a pu porter sur un affinement de la sylviculture, moins carrée sur les chantiers 


\section{LECLERC - Murièle MILLOT}

étudiés que dans les "coupes de lisières" de futaie régulière ordinairement pratiquées dans cette vallée du Trentin. Dans ces trois cas, le bilan économique est resté largement positif.

En France, ce sont plutôt les modes d'exploitation "nouveaux" qui ont été promus : plus précisément le câble dans diverses variantes, notamment en combinaison avec d'autres techniques, le tracteur aux Contamines, ou l'hélicoptère à Abondance. Dans un contexte où domine une forte culture de jardinage pied à pied, cela a conduit à adapter la sylviculture dans le sens d'une relative concentration des prélèvements sous forme de trouées.

Ces infléchissements de sylviculture, presque opposés entre les deux pays, convergent vers un concept de plus en plus partagé à l'échelle de l'arc alpin : une sylviculture par groupes d'arbres, respectant la structure naturelle des forêts d'altitude, ou cherchant à l'imiter à plus basse altitude dans un souci de stabilité, de diversité et d'économies.

Au-delà des aspects techniques, l'étude a fait également apparaître la force des habitudes et des cadres réglementaires susceptibles d'entraver les évolutions, notamment en France : habitude de la vente sur pied, exploitations en bois long, par tracteur plutôt que par câble, subventions à la création de routes plutôt qu'à l'exploitation, régime de TVA... L'échec d'Abondance, les difficultés rencontrées à Pralognan sont aussi significatives de blocages au niveau des municipalités, pas toujours encouragées à gérer le long terme.

$\mathrm{Si}$, dans beaucoup de cas, les techniques et structures actuelles sont suffisantes pour gérer la forêt de montagne avec une réelle finesse et une efficacité économique certaine, elles sont cependant défaillantes pour des cas sortant de la norme. Or ces cas seront de plus en plus nombreux, à mesure que seront mieux exprimées les attentes, notamment en matière de protection de la nature et des paysages, et que se diversifieront les techniques. À terme, c'est l'idée même de norme qui peut se trouver condamnée.

C'est pourquoi, pour aller au-devant de blocages de grande ampleur comme ceux déjà constatés en Suisse ou en Allemagne, il est important de poursuivre une politique d'innovation, sur la base de chantiers pilotes comme ceux présentés dans le présent article. Ces chantiers sont appelés à former un réseau de références technico-économiques pour alimenter la réflexion socio-politique : l'imagination et l'initiative sont à mobiliser au niveau des techniques forestières, comme à celui des modes de financement, à réorienter en partie vers l'aide à l'exploitation "de précision".

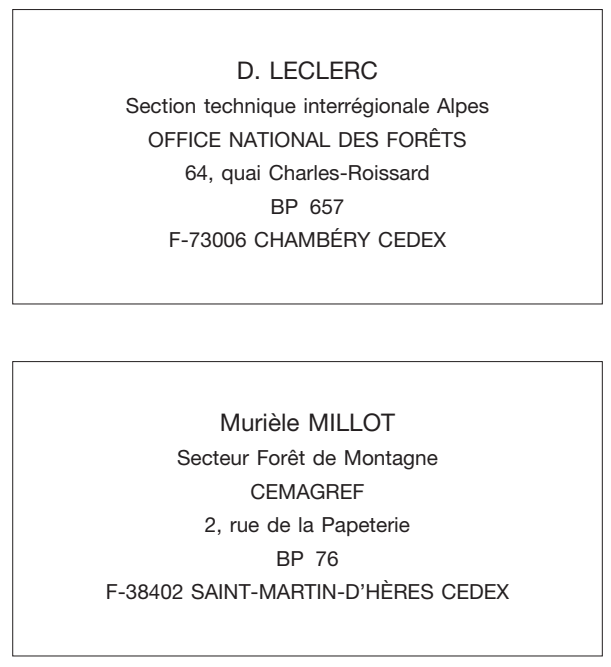




\section{BIBLIOGRAPHIE}

BIANCHI (M.), POLLINI (C.), SPINELLI (R.), TOSI (V.). - Mountain forests : technics for long-term multi-functional management. - Rapport final italien, 1998. - 14 p. (EU Project LIFE/94/F/A15/EU/00713).

CHAUVIN (C.). - Forêts de montagne : techniques pour une gestion multifonctionnelle durable. Alpes françaises et italiennes. - Grenoble : CEMAGREF - Division EPM - Équipe Forêt, 1994. - 23 p. (Document technique) (EU Project LIFE/94/F/A15/EU/00713).

MERMIN (E.). - Forêts de montagne : techniques pour une gestion multifonctionnelle durable - Alpes françaises et italiennes. Résultats de l'analyse et des inventaires. Diagnostic et interventions sylvicoles: forêt communale d'Abondance. - Grenoble : CEMAGREF - Division EPM - Equipe Forêt, 1997. - 13 p.

MERMIN (E.), MILLOT (M.). — Forêt communale d'Abondance. — Grenoble : CEMAGREF - Division EPM - Équipe Forêt, 1998. - 19 p.

MERMIN (E.), MILLOT (M.). - Forêt communale des Contamines-Montjoie. — Grenoble : CEMAGREF - Division EPM Équipe Forêt, 1998. - 8 p.

MILLOT (M.). - Forêt communale de Pralognan-la-Vanoise. - Grenoble : CEMAGREF - Division EPM - Équipe Forêt, 1998. - $12 \mathrm{p}$.

MILLOT (M.). - Forêt domaniale du Boutat. - Grenoble : CEMAGREF - Division EPM - Équipe Forêt, 1998. - 16 p.

PISCHEDDA (D.), CHAGNON (J.-L.). — Site du Boutat : choix d'un système d'exploitation. — Paris : CTBA, 1998. — 3 p.

PISCHEDDA (D.), CHAGNON (J.-L.). — Site de Pralognan-la-Vanoise : stratégie globale d'intervention. - Paris : CTBA, 1998. $-5 \mathrm{p}$.

RENAUD (J.-P.), MERMIN (E.), PEILLEIX (A.). — Forêts de montagne : techniques pour une gestion multifonctionnelle durable - Alpes françaises et italiennes. Résultats de l'analyse et des inventaires. Diagnostic et interventions sylvicoles: forêt domaniale du Boutat (Isère) et forêt communale de Pralognan-la-Vanoise (Savoie). - Grenoble : CEMAGREF - Division EPM - Équipe Forêt, 1996. - 30 p.

TOSI (V.). - Résumé de "Mountain forests : technics for long-term multi-functional management". — Rapport final italien, 1998. - 2 p. (EU Project LIFE/94/F/A15/EU/00713).

\section{TECHNIQUES POUR UNE GESTION MULTIFONCTIONNELLE DURABLE DES FORÊTS DE MONTAGNE : CAS DES ALPES EN FRANCE ET EN ITALIE (Résumé)}

Dans les forêts de montagne qui doivent remplir de nombreuses fonctions (protection physique, production, patrimoine, écologie...), les interventions sylvicoles préconisées pour y parvenir ne sont pas toujours réalisables pour des raisons économiques.

Une étude sur cette gestion multifonctionnelle a été réalisée sur 6 forêts de montagne ( 3 en France et 3 en Italie). Elle a permis de combiner un diagnostic sylvicole et un diagnostic d'exploitation par site, de choisir le meilleur compromis et de réaliser les travaux de récolte retenus.

Les bilans technico-financiers sont très variables d'un site à l'autre et montrent qu'il n'y a pas de méthode unique. Seule une analyse cas par cas permet de trouver la solution la plus adaptée. Si les concepts en matière de sylviculture de protection dans l'Arc alpin convergent de plus en plus, les conditions économiques divergent encore sensiblement et nécessitent de faire preuve de beaucoup d'esprit d'innovation.

\section{TECHNIQUES FOR A SUSTAINABLE, MULTIFUNCTIONAL MANAGEMENT SYSTEM FOR MOUNTAIN FORESTS : THE CASE OF THE FRENCH AND ITALIAN ALPS (Abstract)}

Recommended sylvicultural practices are not always economically feasible in mountain forests where a whole range of functions must be fulfilled (physical protection, production, heritage, environment, etc.).

A study of multifunctional management was conducted on six mountain forests (three in France and three in Italy). Sylvicultural and logging considerations were both evaluated for each individual location, based on which the best compromise and consequent felling schemes were chosen and carried out.

The technical and financial results vary considerably from one site to another showing that there can be no one universally applicable method. Finding most suitable solution necessarily requires each case to be considered individually. Concepts in the area of protective sylviculture are converging more and more in the Alpine countries, but economic conditions are still significantly different. As such, a highly innovative spirit is needed. 